\title{
Acceptability of Health Information Exchange and Patient Portal Use in Depression Care Among Underrepresented Patients
}

\author{
Elizabeth B. Matthews, PhD, LMSW' (1), Margot Savoy, MD, MPH 2,3, \\ Anuradha Paranjape, $M D, M P H, F A C P^{2}$, Diana Washington, $B A^{3}$, \\ Treanna Hackney, $B A^{4}$, Danielle Galis, $B A^{7}$, and Yaara Zisman-llani, PhD, $M A^{4,5}$
}

'Graduate School of Social Service, Fordham University, New York, NY, USA; ${ }^{2}$ Lewis Katz School of Medicine, Temple University, Philadelphia, PA, USA; ${ }^{3}$ American Academy of Family Physicians, Washington, DC, USA; ${ }^{4}$ College of Public Health, Temple University, Philadelphia, PA, USA; ${ }^{5}$ Division of Psychology and Language Sciences, Department of Clinical, Educational and Health Psychology, University College London, London, UK.

BACKGROUND: Depression is often untreated or undertreated, particularly among underrepresented groups, such as racial and ethnic minorities, and individuals of lower socioeconomic status. Electronic health information exchange (HIE) is a recommended practice to improve care coordination and encourage patient engagement in services, but it remains underutilized in depression care. Understanding factors affecting acceptance and adoption of this technology among underrepresented patient populations is needed to increase dissemination of HIE within mental health treatment.

OBJECTIVE: The present study aims to identify patient barriers and facilitators towards the acceptance of HIE within the context of depression treatment and to examine how HIE impacts depression-related care coordination and patient activation.

DESIGN: Semi-structured qualitative interviews were conducted with 27 patients.

PARTICIPANTS: Respondents were English-speaking adults ( $>18$ ) receiving depression treatment within a large, safety-net primary care clinic.

APPROACH: A grounded theory approach was used to code and analyze data for emergent themes. Thematic analysis was guided by the Unified Theory of Acceptance and Use of Technology, a leading informatics theory used to predict end-user adoption of technology.

KEY RESULTS: Respondents reported that HIE made depression care more convenient, transparent, and trustworthy. Though respondents desired greater access to their health records, stigma surrounding depression inhibited acceptance of electronic communication and information sharing. Confusing electronic interface also diminished perceived benefits of HIE.

CONCLUSION(S): Respondents desire greater transparency in their depression care. While HIE was perceived to improve the overall quality of depression care, stigma associated with mental illness undermined more robust adoption of this technology among underserved populations.

KEY WORDS: Health information exchange; Patient portals; Unified Theory of Acceptance and Use of Technology; Depression; Health disparities.

Received October 5, 2021

Accepted January 19, 2022

Published online February 7, 2022
J Gen Intern Med 37(15):3947-55

DOI: $10.1007 / \mathrm{s} 11606-022-07427-2$

(c) The Author(s) under exclusive licence to Society of General Internal Medicine 2022

\section{BACKGROUND AND RATIONALE}

Depression is a leading cause of disability ${ }^{1}$ but is often untreated or undertreated, especially among medically underserved patients, such as those from racial and ethnic minorities or in lower socioeconomic brackets. ${ }^{2,3}$ Currently, as few as $35.7 \%$ of individuals with newly diagnosed depression initiate treatment. $^{2}$ Among those engaged in care, less than $25 \%$ receive care that met recommended standards ${ }^{4}$ and many leave treatment earlier than recommended. ${ }^{5,62,37}$ In response to these challenges, efforts have largely focused on improving initial service linkages through systematic coordination within and across health and mental health care systems, ${ }^{8-10}$ and encouraging sustained engagement in services by keeping individuals actively involved in their depression care. ${ }^{1-14}$

Care coordination and patient activation efforts rely on provider's ability to effectively communicate and exchange information with both patients and other professionals. This capacity is largely dependent on successful utilization of health information exchange (HIE), where patient health information is stored, retrieved, and updated via transmitted electronic health records (EHRs). ${ }^{15}$ HIE refers to electronic functionality that "allows doctors, nurses, pharmacists, other health care providers and patients to appropriately access and securely share a patient's vital medical information electronically, improving the speed, quality, safety and cost of patient care". ${ }^{16}$ HIE can be provider-facing (i.e., directed- or querybased exchange) or patient-facing (i.e., patient-mediated exchange). ${ }^{16,17}$ Providers use HIE to better coordinate care, facilitate service linkages, and exchange relevant health information so that decisions about health care can be made using the most accurate and complete information. Patient-facing HIE primarily occurs using patient portals and personal health records, which allow individuals to communicate directly with 
providers and access their health record, such as medication, lab results, and visit notes.

Both forms of HIE present opportunities to improve engagement in depression treatment. Provider HIE can be used to identify high-risk individuals in need of depression care, ${ }^{18}$ increase the frequency of communication among the treatment team members, and improve engagement in mental health services. ${ }^{19}$ Patients' use of portals and personal health records has similarly been associated with higher rates of treatment completion and symptom remission from depression ${ }^{20}$ and increases in patient-rated involvement in care. ${ }^{21,22}$ Despite these benefits, widespread adoption of both provider $\mathrm{HIE}^{23,24}$ and patient portals ${ }^{25-27}$ has been slower than anticipated, particularly among racial and ethnic minorities and those with lower educational attainment, ${ }^{24,26,28,29}$ groups that are also at higher risk for untreated or undertreated depression.

Existing work has largely focused on identifying providerand system-level factors impacting both provider HIE and patient portal use, concluding that persistent barriers include poor functional design (i.e., user-friendliness) of HIE platforms, and concerns about information accuracy and security. ${ }^{23,24}$ Patient-reported barriers, however, have been far less investigated, and virtually none of this work has examined factors impacting patient acceptance of provider HIE or portal use within the context of depression care.

To optimize the potential of HIE to improve depression care, targeted research exploring barriers and facilitators to patients' acceptance of this technology within the context of depression treatment is needed. The Unified Theory of Acceptance and Use of Technology (UTAUT), ${ }^{30,31}$ a leading implementation framework in the field of informatics, articulates key conditions impacting end-user acceptance and utilization of new technology. According to the UTAUT, acceptance is a necessary precondition to adoption of a new technology and is shaped by the end users' expectation that the technology will make targeted tasks easier (i.e., performance expectancy), will be easy to use (i.e., effort expectancy), and is accepted by other members of their social group (i.e., social influence). Refinements to the UTAUT include the role of facilitating conditions, or the presence of knowledge and concrete resources necessary to use a technology, in predicting acceptance. ${ }^{31}$ UTAUT has been used to guide implementation strategies to increase provider acceptance and adoption of technology in a number of health settings, ${ }^{32-34}$ but has yet to be systematically applied to understand the barriers or facilitators affecting patients' adoption of HIE in mental health. Guided by the UTAUT framework, the aims of this qualitative investigation were to (1) identify patient barriers and facilitators towards the acceptance of HIE within the context of depression primary care and (2) examine how patients perceived HIE to impact depression-related care coordination and patient activation.

\section{Setting and Participants}

METHODS

The study took place within a large safety-net hospital system serving one of the poorest catchment areas of Philadelphia, PA (North and Lower North City Planning Districts). The patient population reflects traditionally medically underrepresented groups, with the majority being predominantly Black or African American, and more than $70 \%$ receiving Medicare or Medicaid. Recent reports show that this area has the highest rates of all causes of medical mortality and is characterized by disproportionately low socioeconomic status. ${ }^{35-37}$

The hospitals and affiliated ambulatory clinics use Epic, a large commercial EHR system, and the MyChart patient portal, which is available to all individuals receiving care within the system.

Eligible patients were identified using EHR chart data and included English-speaking adults ( $>18$ years) with a diagnosis of depressive disorder as defined by ICD-10-CM diagnostic codes F22 and F33. Recruitment was limited to patients receiving care within the system's two primary care practices, Internal Medicine and Family \& Community Medicine, with at least one primary care visit within the previous 12 months. Using a complete list of eligible participants, recruitment was conducted by three research assistants using a consecutive sampling approach. Because the study was conducted during the COVID-19 pandemic, individuals agreeing to enroll in the study were interviewed over the phone or via video. Interviews with participants were recorded and lasted an average of 45 min (Table 1).

Following established frameworks for qualitative interview guides, ${ }^{38}$ a semi-structured interview guide was developed by the first and last authors using UTAUT constructs and the extant literature on adoption and acceptance of patient and provider HIE (see Appendix I). To best capture the nuances of the participants' experience, the interview was organized around main questions and follow-up prompts, ${ }^{35}$ which are probing questions or responses designed to guide deeper understanding of phenomena of interest. To ensure rigor, the interview guide was piloted internally for comprehensiveness and flow. Three trained research assistants conducted interviews. Respondents received a \$20 gift card for their participation. Study protocols were reviewed and approved by a university institutional review board.

\section{Analysis}

All interviews were transcribed verbatim and uploaded into Dedoose, a qualitative analysis platform. Coding and analysis were guided by contemporary applications of a grounded theory approach. ${ }^{39}$ This inductive method requires a multistage, iterative approach to analysis, beginning with open coding, or a line-by-line examination of interview transcripts designed to identify broad concepts derived from the data. The process of open coding was guided by preestablished 
Table 1 Sample Interview Guide and Summary of Key Themes

\begin{tabular}{l}
\hline Question \\
\hline Keeping information private is an \\
important part of providing good care. \\
How confident are you that computers \\
and electronic health records can keep \\
your information private?
\end{tabular}

\begin{tabular}{|c|c|}
\hline $\begin{array}{l}\text { UTAUT } \\
\text { domain }\end{array}$ & Summary of key theme(s) \\
\hline $\begin{array}{l}\text { Performance } \\
\text { Expectancy }\end{array}$ & $\begin{array}{l}\text { - Respondents were overall confident in } \\
\text { the ability of portals and HIE to keep } \\
\text { information secure from breaches, though } \\
\text { some risk was acknowledged } \\
\text { - Despite this, many were guarded about } \\
\text { which providers would have access to } \\
\text { their mental health information } \\
\text { - Respondents described fear of stigma, } \\
\text { labeling, or unauthorized disclosure of } \\
\text { their mental illness }\end{array}$ \\
\hline
\end{tabular}

Sometimes, doctors' offices have websites or portals that let you access your health information online. Have you ever accessed your health information online?

Probe: Ifyes: how was this helpful Probe:no: what got in the way?

Sometimes, providers use the computer during visits. Often, this is to pull up information about your care, send messages to other providers, send prescriptions and referrals, or print out information about your treatment. How does your provider use the computer to help deliver your treatment?
- Patient portals were used to manage care and remain informed about their treatment - Respondents valued having access to their health and mental health information, and desired mechanisms, like the portal, that made accessing care convenient

- Respondents consistently articulated the value of coordinated care, and the desire for their providers to communicate - HIE between providers was seen as a critical component of delivering high-quality care
Performance Expectancy Effort Expectancy

Performance Expectancy

Effort Expectancy Facilitating Conditions

\section{Sample quotations}

- I'm comfortable with it as long as they have the proper security of course, is secure as anything else to be honest. - But when I was like there was a lot of personal information that comes out during, like, a inpatient stay it gets transferred over to like your medical doctor and your family doctor. I don't think that's necessary.

- What shouldn't be on the computer for the most part anything that you know anything that the person wants to keep very confidential between, like, very little people should know. That's why I don't think it should be in a file on a computer - So if there is a study done um you know and once he releases the test I can see the results....It's very useful because since my memory it's kind of you know confusing and cloudy. If I need to tell another doctor I can go back in there refresh and talk a little bit about it you know, so I don't miss any details.

- Oh gosh um the um what's it called ... [portal] but whatever, the getting prescriptions refilled is super easy

- I think that the sharing within [hospital system] and those doctors. I think is crucial for each one of them to have a way to openly understand what is going on with me.

- When I get a consult for another medical problem. That person reads up on me before I go for the appointment. And then they say to me like oh well this might interfere with that. So, I'm glad that I knew that. Or if I forget to tell them something...So, I think that the more complete the more information in, it the better.

- [family member] will pull up information from my chart...it is better her than me because I don't use the computer

- I'm definitely a tech guy and that's what my fiancé most think about me

- ...the websites or the online portals that are too confusing for me to understand. - I've learned the tricks around it you have to poke around in there until you learn it sensitizing concepts, or "interpretive devices" that provide an organizing framework for making sense of the data. ${ }^{40}$ Often informed by the investigators' basic research questions, sensitizing concepts guiding this study's initial coding included patients' communication preferences, their experience using HIE, and their attitudes towards technology.

Following this initial stage of open coding, a codebook is further refined using a constant comparative method, which refers to an iterative process where similarities and differences between and within codes are systematically examined. ${ }^{39}$ To accomplish this, the first author and a research assistant first independently coded a sub-sample of interviews, then compared codes in order to identify and refine key emergent themes, and resolve any discrepancies through consensus building. To enhance objectivity and triangulate data, the last author, who was not involved in the coding process, was involved in the process of adapting, refining, and distilling codes into more precise constructs. A total of $20 \%$ of transcripts $(n=6)$ were co-coded and reviewed in this manner to establish reliability in coding. This process was repeated until no new themes emerged, and remaining transcripts were coded using the finalized codebook. An audit trail of all team meetings and codebook revisions was kept to further promote rigor.

Inductive thematic analysis ${ }^{41}$ was used to understand key questions about how patient and provider HIE was used within the context of depression care, and the ways in which HIE helped or harmed patients' experience in treatment. To examine any sources of discrepancy, cross-case analysis ${ }^{42}$ was also used to examine differences among the diverse sample. 
Table 2 Sample Demographics $(n=27)$

\begin{tabular}{lll}
\hline \hline & $\boldsymbol{N}$ & $\boldsymbol{\%}$ \\
\hline Sex & & \\
$\quad$ Male & 8 & 30 \\
$\quad$ Female & 19 & 70 \\
Race/ethnicity & & 33 \\
$\quad$ White & 3 & 55 \\
Black/African American & 15 & 6 \\
Non-White Hispanic/Latinx & 1 & 6 \\
Other & 1 & 33 \\
Age (years) & & 19 \\
18-35 & 9 & 33 \\
36-45 & 5 & 11 \\
46-55 & 9 & 33 \\
65+ & 3 & 52 \\
Education & & \\
$\quad$ High school & 9 & \\
Some college & 4 & \\
College degree or more & 14 & \\
\hline
\end{tabular}

Additional strategies to increase the trustworthiness of the themes identified in this data included routine peer debriefing, data triangulation, an audit trail, and negative case analysis. ${ }^{43}$

\section{RESULTS}

Study recruitment continued until the research team achieved a consensus that thematic saturation ${ }^{44}$ was reached, meaning that no new codes or themes were evident from interviews. A total of 314 individuals were contacted for enrollment. Of this, 226 declined, 59 were lost to follow-up after an initial contact, and 29 individuals were successfully enrolled. Technical issues compromised the quality of two participants' responses, for a total sample of 27 patient respondents. Sample demographics are summarized in Table 2.

Respondents described a wide range of ways to electronically share information, including electronic scheduling, exchanging messages with providers, and sending and receiving mental health records between treating providers. While some of these activities extend beyond traditional definitions of HIE, they were included in the study results because of their particular impact on respondents' experience in care.

\section{Performance Expectancy of Patient Portals}

The UTAUT framework asserts that a primary predictor in end-user acceptance of technology is performance expectan$c y$, or the degree to which technology will make tasks easier or more effective. A third $(n=17,36 \%)$ of respondents described ways in which patient portals improved their depression care: Efficient Access to Depression Care. Respondents described elements of portal use that made their access to depression care easier and more efficient. Messaging and scheduling features of the patient portal facilitated quick access to respondents' providers, creating opportunities to overcome accessibility barriers associated with reaching providers by phone:

When I send an email through the system I know someone is going to see it, so um, like, I always get an answer whether it is from a nurse or from the doctor um always get a phone call back like it is definitely calling the office is a nightmare um but using the online like portal has been tremendously helpful.

\begin{abstract}
[Portal] was convenient in some ways and in a way of scheduling through a computer. And for appointment I guess it's convenient in some way because you don't have to wait for the person to answer your call or anybody to write it down. You just see which slots are available
\end{abstract}

A critical component of this theme was respondents' emphasis on the value of convenience and efficiency. Messaging and scheduling features allowed patients to manage their depression care on their own time, rather than on the schedule of their provider or doctor's office.

Information Transparency Is Empowering. Another facilitating factor related to HIE's performance expectancy was the role of information access and transparency. Respondents expressed a strong desire to have access to lab tests, medications, and diagnoses:

[Portals] keep me in the loop about the medications. What you taking this for each medication they talk to me to make sure that I am still using it and how many times I am taking it.

You can go home and read about things, so they trigger um different printouts on your medicine, your diagnosis, your treatment plan whatever. Uh, so there is a lot of information right at your fingertips.

Respondents described that patient HIE helped them stay engaged in their treatment by allowing them to track their own care and remember information that could be important for ongoing health maintenance and symptom reduction, such as the names of medications or one's diagnosis.

\section{Performance Expectancy of Provider HIE}

Almost half ( $n=12,45 \%$ ) described a strong preference for coordinated care and viewed HIE as an effective tool to accomplish this. Respondents expressed that the quality of their care was enhanced when providers shared information with one another. By enabling providers to obtain complete and comprehensive information about their current and historical physical and mental health, provider HIE made respondents feel more confident in their providers' recommendations for treatment. This was particularly true, as illustrated below, 
when patients had co-occurring depression and chronic medical conditions.

Just saying if my therapist and my primary care doctor know what is going on with me within medications. And then I'm a diabetic, so I, you know ... yeah I like it ... It's like a tree...every branch functions well because of the other branch... And that makes me comfortable that you can pull me up from a computer and get my doctor's notes from that doctor that's pertaining to this issue.

I think it [portals] actually makes the interaction better. Because before they would have to keep writing things down... and sometimes papers are missing or whatever. Now they look that they look back and say 'oh in 1988 your hemoglobin was 15'. So, I actually think that it in enhances my care.

They will open my record and they can look at things right away... And what's nice is you don't have to physically saying it out loud. ... if they are going to change medication dosage or something, I know that they did it right then and there. So, that's very helpful to me.

\section{Facilitating Factors Inhibiting Acceptance of Provider HIE}

Within the UTAUT framework, acceptance and adoption are also shaped by the availability of perceived resources and support that promote a particular behavior (i.e., facilitating conditions). In our sample, facilitating conditions emerged as barriers to the acceptance of provide HIE.

Stigma Inhibits HIE Acceptance.. Perceived stigma was associated with ambivalence regarding HIE acceptance, as half of respondents $(n=12,45 \%)$ described concerns about who had access to their information:

Everything that is going on with me, is not always physical, but I am very aware of the effect how my mental, mentality effects my physical body. With pain and nausea, it has even affected my eyesight. That um, I that is fine going through that system. But I don't think anyone else should have access to that, unless they ask me for permissions to have it

This ambivalence offered critical insight into the unique function of provider HIE within the context of depression care. Respondents did not endorse strong concerns about the confidentiality or security of their health information generally. Most participants $(n=21,78 \%)$ either felt completely confident in the system's security or equated the level of security risks with any other online task, such as electronic banking or online shopping:

I'm confident I always find out, I mean yeah, there probably hacker in there. ... I feel really comfortable there is not much you can do about it.

I'm comfortable with it as long as they have the proper security of course, is secure as anything else to be honest.

Instead, concerns about exchanging information related to depression appeared closely linked to the stigma associated with mental illness, and how this information would be used or perceived by other parties with access to their health information:

You know, it's certain things you just don't want people to know about you. You know I remember a long time ago, I went through a phase where I was very depress. And I want to seek help, but I was embarrassed, and I was afraid that somebody would find out that I had some mental issues. And somehow that would give people a negative imprint in me. You know, that they would judge me because I needed help.

Yeah, I think there is things on there that shouldn't be share with other entities. You know uh, like, okay, my workplace doesn't need to know. That of course I'm out on mental stress leave. But, they don't need the details of that. I don't want people looking at me differently, because, and they will.

\section{Effort Expectancy Reduces Patient Portal Acceptability}

A third of the respondents $(n=10,37 \%)$ identified usability and digital literacy as a barrier to accepting patient portals. Usability concerns related to the design of patient portals specifically:

It's really hard to navigate uhh the portal. Uhh I just find it confusing.

There is a lot of links and a lot of things that I don't understand it's kind of overwhelming. 
As well as with technology more generally:

\section{I don't know how to do that [use of the portal]. I'm not good with all this, the computers and stuff.}

\section{DISCUSSION}

The present study, guided by constructs from UTAUT, adds to our limited understanding about factors affecting patients' acceptance of HIE in primary care-based depression treatment. The study also provides insight into the potential of HIE to improve care coordination and patient involvement in their own care, key components of the quality and continuity of depression care. Across both provider and patient-facing HIE, patients described high HIE performance expectancy that facilitated accessible, high-quality care. However, concerns about depression-related stigma were perceived as a barrier undermining respondents' willingness to consent to provider HIE. Negative appraisals of patient portal use were primarily driven by high effort expectancy associated with cumbersome or confusing portal interfaces.

\section{Performance Expectancy Facilitates Acceptability of Patient-Facing and Provider- Facing HIE}

Respondents described positive appraisals of performance expectancy related to both patient-facing HIE and provider HIE. A desire to improve access to mental health information was a common theme when discussing both patient- and provider-facing HIE. In addition to making care more accessible through messaging and scheduling functions, patient portals can empower patients by giving them greater control of their own health information. Similar work suggests that providing access to medications, lab results, and other information is increasingly viewed as a core patient-centered care strategy to support health literacy and illness management. ${ }^{45}$

Respondents were clear about their desire for regular communication and coordination, and endorsed provider HIE as an acceptable and efficient mechanism to accomplish this. Provider HIE was associated with several benefits: improving visit efficiency by centralizing information and reducing the patient's need to frequently repeat the same psychiatric history. In contrast with the management of physical health, recounting one's full psychiatric history often places patients into a position of unwanted or uncomfortable vulnerability, ${ }^{46}$ particularly when such information requires recalling traumatic or distressing life events, highlighting a particular benefit to HIE within the context of behavioral health treatment.

\section{Stigma as a Condition Inhibiting Acceptability of Provider HIE.}

Although provider HIE was associated with improved care quality, respondents also expressed concerns about with whom their sensitive information would be shared. Interestingly, though security concerns are frequently cited as a major obstacle to provider HIE, ${ }^{24,47,48}$ fears of or general information breaches were not the most salient force driving respondents' hesitation around HIE. Because technology changes develop rapidly, this may reflect an attitudinal shift as much more information is now stored electronically.

When confidentiality concerns did arise, they focused specifically on the disclosure of sensitive psychiatric history and depressive symptoms, suggesting that the stigma associated with mental illness can inhibit patients' acceptance of mental health-related HIE. This pattern aligns with prior work indicating that patients are less willing to share health information that is perceived to be sensitive or complex. ${ }^{49}$ These attitudes may be particularly relevant within this study's racially and ethnically diverse sample, as stigma around mental illness remains pervasive within Black and African American communities, ${ }^{50,51}$ and longstanding mistrust of the health system ${ }^{52}$ may amplify concerns about disclosing depressive symptoms. Together, these findings highlight that negative labels and stereotypes associated with depression make HIE within mental health treatment differ from acceptability of HIE for managing physical health conditions. Efforts to modify attitudes towards provider HIE must therefore attend to the particular intersection between depression, confidentiality, and culture that is reflective of the patient population being served.

The tension between expanding access to health information while safeguarding patients against stigma has implications for future policies guiding both patient and provider HIE. In an effort to prioritize the protection of individuals' privacy, current policies allow providers to limit what mental health information is shared with patients, ${ }^{53,54}$ and many states require patients to explicitly opt in, or actively consent, to provider HIE before it can occur. While developed as intended safeguards, these restrictive policies may be misaligned with patients' preferences for greater access to their information, and have the unintended consequence of reducing the exchange of heath information. For example, Apathy and colleagues ${ }^{55}$ found that the administrative and legal burdens of opt-in regulations decreased health organizations' capacity to engage in provider HIE. Similarly, in their study of patient portal use within a safety-net ambulatory clinic, Ancker and colleagues ${ }^{56}$ found significant racial and socioeconomic disparities existed when patients had to opt in to receive a portal account; however, racial differences in portal use disappeared when universal (i.e., opt-out) policies were installed. This study's and other recent findings $^{21,57}$ reporting that patients desire greater access to and control of their health records, suggest the need to reconsider how policies can better prioritize transparency and information exchange in health care, and particularly mental health care. 


\section{Effort Expectancy Inhibited Portal Use}

Respondents expressed concerns with the usability of the patient portal, which inhibited acceptability and adoption of this function. Consistent failures to develop more user-friendly and patient-centered interfaces are a well-documented barrier to end-user adoption across numerous forms of health information technology, including mHealth apps, electronic health record systems, and personal health records. ${ }^{47,58-60}$ As evidenced by this sample of patients from a safety-net setting, portals with complex or confusing digital platforms are likely to discourage use and may disproportionately disincentivize use among underrepresented populations. Indeed, over 100 studies have documented disproportionately lower portal use among racial and ethnic minorities, and individuals with lower educational attainment and lower socioeconomic status, ${ }^{61}$ yet few interventions have been developed to explicitly reduce these disparities. Patients' desire to access their records, and the benefits derived from doing so, present a clear motivation for addressing this gap.

Our findings should be considered in light of the following limitations. First, the findings may not be generalizable to individuals with depression who live in other geographic areas. Also, this research utilized a purposeful sample of patients. Patients who had particularly strong or negative opinions about HIE experiences may have been more likely to join the study. In addition, we had a relatively low response rate, and though the final sample reflected the racial and ethnic diversity of the clinics' patient population, our sample was also highly educated (52\% college educated). This is likely the result of conducting the study during the first wave of the COVID-19 pandemic, as restrictions required virtual recruitment and interviews. This may have biased our sample against those with inconsistent internet or access to technology or those with competing demands, such as childcare. As demographic information was not collected from individuals who declined to participate, we cannot draw conclusions about the representativeness of the study sample or how they compared to those who declined to participate. Finally, our study did not evaluate what type of technology was used to access the portal (e.g., computers versus tablets or mobile devices). Some evidence suggests that members of vulnerable groups, including racial and ethnic minorities and those with lower educational attainment, are more likely to access patient portals through mobile devices $^{62}$ rather than computers. Mobile access has also been associated with overall lower rates of portal use, ${ }^{62}$ perhaps due to unmeasured differences in functionality and usability. Future work in this area should focus on gaining a deeper understanding of patterns of access and how this impacts the user experience.

To conclude, this study highlights several ways in which HIE and portal use can enhance depression care for underserved patients, yet barriers to more robust acceptance of these technologies persist. Results underscore the need for a more user-friendly design, while also adding novel insight into how the unique stigma accompanying mental health may inhibit adoption of information-sharing practices that could ultimately support ongoing efforts to better coordinate depression care and activate patients to remain engaged in services.

Corresponding Author: Elizabeth B. Matthews, PhD, LMSW; Graduate School of Social Service, Fordham University, New York, NY, USA (e-mail: ematthews13@fordham.edu).

Supplementary Information The online version contains supplementary material available at https://doi.org/10.1007/s11606-02207427-2.

Funding This study was funded by the Fordham University Faculty Research Development Fund.

\section{Declarations:}

Conflict of interest: The authors declare that they do not have a conflict of interest.

\section{REFERENCES}

1. Friedrich MJ. Depression Is the Leading Cause of Disability Around the World. JAMA. 2017;317(15):1517-1517.

2. Waitzfelder B, Stewart C, Coleman KJ, et al. Treatment Initiation for New Episodes of Depression in Primary Care Settings. J Gen Intern Med. 2018;33(8):1283-1291.

3. Cummings JR, Ji X, Lally C, Druss BG. Racial and Ethnic Differences in Minimally Adequate Depression Care Among Medicaid-Enrolled Youth. Journal of the American Academy of Child and Adolescent Psychiatry. 2019;58(1):128-138.

4. González HM, Vega WA, Williams DR, et al. Depression care in the United States: too little for too few. Archives of General Psychiatry. 2010;67(1):37-46.

5. Fornaro M, Novello S, Fusco A, et al. Clinical features associated with early drop-out among outpatients with unipolar and bipolar depression. Journal of Psychiatric Research. 2021;136:522-528.

6. Schindler A, Hiller W, Witthöft $\mathbf{M}$. What predicts outcome, response, and drop-out in CBT of depressive adults? a naturalistic study. Behavioural and Cognitive Psychotherapy. 2013;41(3):365-370.

7. Cooper AA, Conklin LR. Dropout from individual psychotherapy for major depression: A meta-analysis of randomized clinical trials. Clinical Psychology Review. 2015;40:57-65.

8. Fortney JC, Pyne JM, Smith JL, et al. Steps for implementing collaborative care programs for depression. Population Health Management. 2009; 12(2):69-79.

9. Young AS, Cohen AN, Chang ET, et al. A clustered controlled trial of the implementation and effectiveness of a medical home to improve health care of people with serious mental illness: study protocol. BMC Health Services Research. 2018;18(1):428.

10. Woltmann E, Grogan-Kaylor A, Perron B, et al. Comparative effectiveness of collaborative chronic care models for mental health conditions across primary, specialty, and behavioral health care settings: systematic review and meta-analysis. American Journal of Psychiatry. 2012;169(8):790-804.

11. Alegria M, Nakash $\mathbf{O}$, Johnson K, et al. Effectiveness of the DECIDE Interventions on Shared Decision Making and Perceived Quality of Care in Behavioral Health With Multicultural Patients: A Randomized Clinical Trial. JAMA Psychiatry. 2018;75(4):325-335

12. Funderburk JS, Pigeon WR, Shepardson RL, Maisto SA. Brief behavioral activation intervention for depressive symptoms: Patient satisfaction, acceptability, engagement, and treatment response. Psychological Services. 2020;17(4):443-451. 
13. Matthews EB, Savoy M, Paranjape A, et al. Shared Decision Making in Primary Care Based Depression Treatment: Communication and Decision-Making Preferences Among an Underserved Patient Population. Frontiers in Psychiatry. 2021;12:681165.

14. Zisman-Ilani Y, Roth RM, Mistler, LA. Time to support extensive implementation of shared decision making in psychiatry. JAMA Psychiatry. 2021;78(11):1183-1184.

15. The Office of the National Coordinator for Health Information Technology (ONC). Office-based Physician Electronic Health Record Adoption,. Health IT Quick-Stat \#50 January 2019. https://www.healthit.gov/data/quickstats/office-based-physician-electronic-health-record-adoption. Accessed August 24, 2021.

16. The Office of the National Coordinator for Health Information Technology (ONC). Health Information Exchange October 2019; https:/ /www.healthit. gov/topic/health-it-and-health-information-exchange-basics/health-information-exchange. Accessed August 24, 2021

17. Patel VN, Dhopeshwarkar RV, Edwards A, et al. Low-income, ethnically diverse consumers' perspective on health information exchange and personal health records. Informatics for Health \& Social Care. 2011;36(4):233-252

18. Kasthurirathne SN, Biondich PG, Grannis SJ, Purkayastha S, Vest JR, Jones JF. Identification of patients in need of advanced care for depression using data extracted from a statewide health information exchange: A machine learning approach. Journal of Medical Internet Research. 2019;21(7).e13809.

19. Falconer E, Kho D, Docherty JP. Use of technology for care coordination initiatives for patients with mental health issues: a systematic literature review. Neuropsychiatric Disabilities and Treatment. 2018;14:2337-2349.

20. Pecina J, North F, Williams MD, Angstman KB. Use of an on-line patient portal in a depression collaborative care management program. Journal of Affective Disorders. 2017;208:1-5.

21. Denneson LM, Chen JI, Pisciotta M, et al. Patients' Positive and Negative Responses to Reading Mental Health Clinical Notes Online. Psychiatric Services. 2018;69(5):593-596.

22. Denneson LM, Cromer R, Williams HB, Pisciotta M, Dobscha SK. A qualitative analysis of how online access to mental health notes is changing clinician perceptions of power and the therapeutic relationship. Journal of Medical Internet Research. 2017;19(6):e208.

23. Wang $\mathbf{J Y}$, Ho $\mathbf{H Y}$, Chen JD, et al. Attitudes toward inter-hospital electronic patient record exchange: discrepancies among physicians, medical record staff, and patients. BMC Health Services Research $2015 ; 15: 264$.

24. Eden KB, Totten AM, Kassakian SZ, et al. Barriers and facilitators to exchanging health information: a systematic review. International Journal Medical Informatics. 2016;88:44-51

25. Strudwick G, Clark C, Sanches M, Strauss J. Predictors of Mental Health Professionals' Perceptions of Patient Portals. AMIA Annual Symposium proceedings AMIA Symposium. 2018;2018:989-997.

26. Nambisan P. Factors that impact Patient Web Portal Readiness (PWPR) among the underserved. International Journal Medical Informatics. 2017;102:62-70.

27. Tulu B, Trudel J, Strong DM, et al. Patient Portals: An Underused Resource for Improving Patient Engagement. Chest. 2016;149(1):272 277.

28. Graetz I, Gordon N, Fung V, et al. The Digital Divide and Patient Portals: Internet Access Explained Differences in Patient Portal Use for Secure Messaging by Age, Race, and Income. Medical Care. 2016;54(8):772-779.

29. Garrido T, Kanter M, Meng D, et al. Race/ethnicity, personal health record access, and quality of care. The American Journal of Managed Care. 2015;21(2):e103-113.

30. Venkatesh V, Morris MG, Davis GB, Davis FD. User Acceptance of Information Technology: Toward a Unified View. MIS Quarterly. 2003;27(3):425-478.

31. Venkatesh V, Thong JYL, Xu X. Consumer Acceptance and Use of Information Technology: Extending the Unified Theory of Acceptance and Use of Technology. MIS Quarterly. 2012;36(1):157-178.

32. Dabliz R, Poon SK, Ritchie A, et al. Usability evaluation of an integrated electronic medication management system implemented in an oncology setting using the unified theory of acceptance and use of technology. BMC Medical Informatics \& Decision Making. 2021;21(1):1-11.

33. Fiona W, Kanika ID, Nikolaos M. A mixed-method service evaluation of health information exchange in England: technology acceptance and barriers and facilitators to adoption. BMC Health Services Research. 2021;21(1):1-13
34. Luyten J, Marneffe W. Examining the acceptance of an integrated Electronic Health Records system: Insights from a repeated crosssectional design. International Journal of Medical Informatics. 2021;150.

35. Ouyang M, Siegel C, Washington R. 2014 Vital Statistics Report Philadelphia. Philadelphia; 2017.

36. Gujar V, Siegel C, Washington R. Vital Statistics Report Philadelphia: 2015.; 2018. https://www.phila.gov/media/20181105161054/2015_Vital Statistics_Report.pdf. Accessed December 30, 2018.

37. Philadelphia Department of Public Health. Community Health Assessment

38. Kallio H, Pietilä AM, Johnson M, Kangasniemi M. Systematic methodological review: developing a framework for a qualitative semistructured interview guide. Journal of Advanced Nursing. 2016;72(12):2954-2965.

39. LaRossa R. Grounded Theory Methods and Qualitative Family Research. Journal of Marriage and Family. 2005;67(4):837-857.

40. Bowen GA. Grounded Theory and Sensitizing Concepts. International Journal of Qualitative Methods. 2006;5(3):12-23.

41. Boyatzis RE. Transforming qualitative information: thematic analysis and code development. Sage Publications; 1998.

42. Khan S., VanWynsberghe R. Cultivating the Under-Mined: Cross-Case Analysis as Knowledge Mobilization. Forum: Qualitative Social Research. 2008;9(1):Art. 34.

43. Shenton AK. Strategies for ensuring trustworthiness in qualitative research projects. Education for Information. 2004;22(2):63-75.

44. Saunders B, Sim J, Kingstone T, et al. Saturation in qualitative research: exploring its conceptualization and operationalization. Quality \& Quantity. 2018;52(4):1893-1907.

45. Salyers, MP, Zisman-Ilani Y. Shared decision-making and self-directed care. In: The Palgrave handbook of American mental health policy (pp. 197-228). Palgrave Macmillan, Cham; 2020:197-228.

46. Matthews EB, Savoy M, Paranjape A, et al. Shared decision making in primary care based depression treatment: Communication and decisionmaking preferences among an underserved patient population. Front. Psychiatry. 2021;12;681165.

47. Powell KR. Patient-Perceived Facilitators of and Barriers to Electronic Portal Use: A Systematic Review. Computers, Informatics, Nursing : CIN. 2017;35(11):565-573.

48. Zwaanswijk M, Verheij RA, Wiesman FJ, Friele RD. Benefits and problems of electronic information exchange as perceived by health care professionals: an interview study. BMC Health Services Research. 2011;11:256

49. Serrano KJ, Yu M, Riley WT, et al. Willingness to Exchange Health Information via Mobile Devices: Findings From a Population-Based Survey. Annals of Family Medicine. 2016;14(1):34-40.

50. Cooper LA, Gonzales JJ, Gallo JJ, et al. The acceptability of treatment for depression among African-American, Hispanic, and white primary care patients. Medical Care. 2003;41(4):479-489.

51. Menke R, Flynn H. Relationships between stigma, depression, and treatment in white and African American primary care patients. Journal Nervous and Mental Disease. 2009;197(6):407-411.

52. Jaiswal J. Whose responsibility is it to dismantle medical mistrust? Future directions for researchers and health care providers. Behavioral Medicine. 2019;45(2):188-196.

53. Blease C, Salmi L, Rexhepi H, et al. Patients, clinicians and open notes: information blocking as a case of epistemic injustice. Journal of Medical Ethics. Epub 24 May 2021.

54. O'Neill S, Blease C, Delbanco T. Open Notes Become Law: A Challenge for Mental Health Practice. Psychiatric Services. 202 1;72(7):750-751.

55. Apathy NC, Holmgren AJ. Opt-in consent policies: potential barriers to hospital health information exchange. Am J Manag Care. 2020;26(1):e14-e20.

56. Ancker JS, Nosal S, Hauser D, et al. Access policy and the digital divide in patient access to medical records. Health Policy and Technology. 2017;6(1):3-11.

57. Bernaerdt J, Moerenhout T, Devisch I. Vulnerable patients' attitudes towards sharing medical data and granular control in patient portal systems: an interview study. Journal of Evaluation in Clinical Practice. 2021;27(2):429.

58. Marzano L, Bardill A, Fields B, et al. The application of mHealth to mental health: opportunities and challenges. The lancet Psychiatry. 2015;2(10):942-948

59. Terry NP, Francis LP. Ensuring the Privacy and Confidentiality of Electronic Health Records. University of Illinois Law Review. 2007;2007(2):681-736 
60. Ratwani RM, Hodgkins M, Bates DW. Improving Electronic Health Record Usability and Safety Requires Transparency. JAMA. 2018;320(24):2533-2534.

61. Grossman LV, Masterson Creber RM, Benda NC, et al. Interventions to increase patient portal use in vulnerable populations: A systematic review. J Am Med Inform Assoc. 2019;26(8-9):855-870.
62. Graetz I, Huang $\mathbf{J}$, Brand $\mathbf{R J}$, et al. Bridging the digital divide: Mobile access to personal health records among patients with diabetes. Am J Manag Care. 2018;24(1):43-48.

Publisher's Note: Springer Nature remains neutral with regard to jurisdictional claims in published maps and institutional affiliations. 